\title{
Artikel
}

Solvejg Nitzke \& Lars Koch*

\section{Prekäre Heimat. Programmatik und Scheitern eines Entstörungsversuchs}


Abstract: ,Heimat' hat nicht nur Konjunktur, sondern erlebt geradezu eine Renaissance. Bemerkenswert ist an ihr jedoch nicht allein die Vielzahl und Lautstärke der Forderungen nach "Umdeutung" oder "Neuausrichtung" des Konzepts, sondern auch der Gestus der Aufklärung und Rehabili-tation, der das programmatisch Nicht-Neue des Ausdrucks zur Kernforderung einer konserva-tiven Rekalibrierung nicht nur gesellschaftlicher, sondern auch gesellschafts- und kulturwis-senschaftlicher Verhältnisse macht. Die damit versuchte Reartikulation von ,Heimat' besteht in der Bekräftigung und Akzeptanz ,natürlicher' Zugehörigkeit, mag sie lokal, regional oder global skaliert sein. Angesichts eines weltweit geführten Diskurses über Prekaritätserfahrun-gen und Zukunftsunsicherheit mag die Rückbesinnung auf einbettende Naturalisierungsstrate-gien dieser Art nicht überraschen, aus kulturwissenschaftlicher Perspektive aber muss sie beun-ruhigen. Es handelt sich nämlich nicht um die harmlose Rückbesinnung auf Tradition(en), sondern legt einen Naturalisierungswillen offen, dessen normative Macht nicht unterschätzt werden darf. ,Heimat' mit den Mitteln und Methoden der Kulturwissenschaften zu beobach-ten und in seinen normativen Implikationen zu beschreiben ist im gegenwärtigen politischen Klima unbedingt geboten. Allerdings kann diese Auseinandersetzung nur dann Wirkung zeigen, wenn sie ihre Bedingungen und Strategien offenlegt. Das Sonderheft „Prekäre Heimat" versammelt Störfälle ,natürlicher' Zugehörigkeit und fragt nach den Verfahren, den Orten, den Diskursen und Akteuren dieser Produktion von Naturverhältnis und Wirklichkeit. Im ein-leitenden Beitrag diskutieren Solvejg Nitzke und Lars Koch die Programmatik und das Scheitern von "Heimat" als Entstörungsversuch und eröffnen so eine Sammlung von Untersuchungen von ,prekärer Heimat', die von Dinosauriern und Urmenschen bis zu Außerirdischen, von Deutschland und Österreich über Frankreich, Ghana, Zimbabwe, Senegal, die Vereinigten Staaten bis zum Blick auf den Planeten Erde reicht.

'Heimat' is booming to a degree that can rightly be called a renaissance. Noticeable is not only the variety of claims to "readjust" and "reframe" the concept, but its role within a conservative attempt to recalibrate societal conditions as well as the circumstances of the humanities and cultural studies. The 'not-newness' of the expression, thus, forms the core of an attitude of enlightenment and rehabilitation which attempts to rearticulate 'Heimat' in order to affirm a sense of 'natural' belonging, whether on a local, regional or global scale. In view of a global debate about experiences of precarity and insecurity towards the future, the return to strate-gies of naturalization and embeddedness may not be all too surprising, but from a cultural studies perspective it is no less unsettling. It is by no means a harmless reactivation of tradi-tion(s), but unveils a will to naturalization whose normative force must not be underestimated. The current political climate calls for the cultural studies to observe and describe 'Heimat' using methods and media to analyze and account for its normative implications. However, this examination can only show the desired effects if it makes transparent its own conditions and strategies. This special issue on "precarious Heimat" assembles disruptions of 'natural' belonging and inquires after the techniques, the places, the discourses and actors of this particular way of producing human-nature-relationships and reality. In their opening essay, Solvejg Nitzke and Lars Koch discuss the objectives and failures of 'Heimat' as a device of renormali-zation and disruption management, thus opening the floor for a collection of 'Heimat'-analyses, which spans dinosaurs, primordial humans and extraterrestrial beings; covers Germany, Austria, France, Ghana, Zimbabwe, Senegal and the United States of America and even a view of the whole Earth.

Keywords: Heimat, Störung, Natur, Naturalisierung, Kulturkritik, Kulturwissenschaft, Herkunft

\footnotetext{
*Dr. Solvejg Nitzke, TU Dresden, Institut für Germanistik, Professur für Medienwissenschaft und NdL, E-Mail: Solvejg.Nitzke@tu-dresden.de

Prof. Dr. Lars Koch, TU Dresden, Institut für Germanistik, Professur für Medienwissenschaft und NdL, E-Mail: Lars.Koch@tu.dresden.de
} 
Den Deutschen Buchpreis 2019 hat ein Nicht-Heimatbuch gewonnen. Keine Anti-Heimat-, keine Heimaterzählung, sondern Saša Stanišićs Herkunft. ${ }^{1}$ Der autofiktionale Text handelt, so Stanišić, von "meinen Heimaten"; von Višegrad, Heidelberg, Verwandten, Verwandtschaftsgeschichten, von Ess-Kulturen, Sprachen und Spracherwerb, Dialekten, Soziolekten und den unterschiedlichen Weisen, in denen sich Menschen verschiedener Generationen an unterschiedlichen Orten einleben. Die Geschichte, die Herkunft erzählt, hat alle Merkmale einer "gelungenen" Integration, die der Preis als eine Art Peritext krönt. In dieser Geschlossenheit lässt sich das jedoch nur dann behaupten, wenn man ignoriert, dass sich Stanišićs Text solcher Geschlossenheit vehement verweigert. Weder lässt sich eine besondere Nostalgie für Višegrad feststellen, noch eine "Verwurzelung" in der ,neuen Heimat'. Es gibt Orte, Menschen und Dinge, die besondere Bedeutung in der Erzählung erlangen, doch nichts wird zur ,Heimat' im emphatischen Sinne, nichts gewinnt den Rang einer ,natürlichen' Zugehörigkeit. Beziehungen sind hier ebenso gewählt und pflegebedürftig, wie die Wohnorte und die Sprache, die gesprochen wird. Dass ein paradigmatischer Nicht-Ort eine Tankstelle in Heidelberg - den Mittelpunkt von ,Sašas' Teenagerleben bildet, ist ein beinahe schon zu guter Twist. Herkunft handelt vom Zufall der Geburt, von der Kontingenz all dessen, was danach kommt und von der Arbeit, die es erfordert, daraus ein Leben, gar eine Erzählung zu machen.

So weit so gut, so konventionell. Was den Text besonders eignet, um an ihm Prekarität von ,Heimat' zu zeigen, ist seine spielerische Haltung zu Wohnort, Integration und Spracherwerb als Naturalisierungsinstrumenten. Die Abwesenheit von Schicksal und der Plural von ,Heimat' lösen weder Nostalgie noch Weltschmerz, weder Frustration, noch Bedauern aus, vielmehr bilden Kontingenz und Fragmentierung hier das Material für die Gestaltung einer eigenen Geschichte, die nicht vorgeprägt, sondern kontingentes Ergebnis einer aktiven Verknüpfungsarbeit ist. Nicht umsonst ist der letzte Teil des Buchs ein Spiel: Bestandteile einer Geschichte sind nacheinan-

1 Dieses Vorhaben wird unterstützt aus Mitteln des Zukunftskonzepts der TU Dresden, finanziert aus Mitteln der Exzellenzinitiative des Bundes und der Länder. der abgedruckt, die Reihenfolge wählt die*der Leser*in selbst, indem er*sie sich am Ende eines Absatzes für eine der angebotenen Alternativen, wie es weitergeht, entscheidet. Dass es sich hier um ein "choose-your-own-adventure"Spielbuch, ein populäres Format für Kinder und Jugendliche handelt, verhindert, dass Herkunft zur x-ten Heimatgeschichte nach Schema F wird: auf Heimatverlust folgen -sehnsucht und -suche, schließlich Heimat(neu)gründung oder Tod. ${ }^{2}$ Ganz im Gegenteil, als Nicht-Heimatgeschichte positioniert sich Herkunft geschickt zwischen Pro- und Anti-Heimat-Literatur. Dadurch wird ,Heimat' in all seiner konzeptuellen Unzulänglichkeit sichtbar, ohne dass die affektive und emotionale Energie dieses Ausdrucks geleugnet würde. Anstatt sich am Unerreichbaren aufzureiben, jeden Schritt mit Pathos aufzuladen und sich selbst zum Exempel der Migrationsgeschichte zu machen, experimentiert dieser Text mit der Möglichkeit, Zugehörigkeit jenseits von ,Heimat' und ,Natürlichkeit' zu erzählen. So weit scheint der nicht-literarische Diskurs noch nicht - oder gerade: nicht mehr zu sein.

\section{1 ,Heimat' in Anführungszeichen}

Texte über ,Heimat', ebenso wie solche über das anscheinend unaufhaltsame Interesse an Texten über ,Heimat' beginnen oft mit ostentativer Überraschung: entweder über die Tatsache, dass der Ausdruck sich so großer Beliebtheit erfreut oder darüber, dass er auf so viel Widerstand trifft. Sie gehen dann die verschiedenen Bedeutungen durch, entwickeln eine Begriffsgeschichte und identifizieren in vielen Fällen einen ,Heimatbegriff zu dem hin oder zurück gearbeitet werden müsse. Ein Merkmal der Debatte ist Unschärfe bei gleichzeitiger diffuser Letztbegründungsverheißung - nicht etwa, weil es an Definitionsversuchen mangeln würde, sondern weil der Wirklichkeitsgehalt des Ausdrucks so prekär ist, dass nie ganz klar wird, ob nun über einen Ort, einen Raum, ein Gefühl, eine Idee, ein Konzept oder gar über einen Begriff gesprochen wird.

2 Paradigmatisch durchgespielt findet sich dieses populäre Erzählschema im erfolgreichen Kinofilm Almanya - Willkommen in Deutschland (R: Yasemin Sandereli, BRD 2011). Vgl. hierzu Koch 2013. 
Trotz des universalen und universalisierten Anspruchs, der ,Heimat' zugeschrieben wird, gibt es in den Wissenschaften nach wie vor keinen "allgemeinen Begriff" (vgl. Penke 2014, 109). Allein aus den von uns herangezogenen Texten lassen sich 22 Definitionen nach dem Muster "Heimat ist..." finden. Wenn man die syntaktische Folge unbeachtet lässt finden sich in den gleichen Texten mehr als fünfzig verschiedene Definitionen: Demnach „ist" ,Heimat' "ein Menschenrecht, Nation nicht. Heimat ist konkret, Nation ist abstrakt" (Menasse 2012, 80); "ein natürliches und ursprüngliches Bedürfnis der Menschen" (Costadura/Ries/Wiesenfeld 2019, 18) ${ }^{3}$; "Heimat ist die Hoffnung und das Versprechen eine Resonanzbeziehung zur Welt einzugehen" (Rosa 2019, 153) und ein "zentraler Identitätsbegriff, der über die Trias von Gemeinschaft, Raum und Tradition eine geschlossene, heile Welt' entwirft, die eine Befriedigung der fundamentalen Bedürfnisse nach Identität und Sicherheit in Aussicht stellt" (Penke 2014, 109). Armin Nassehi bestimmt Heimat als „Ersatzhandlung. Wofür, dürfte inzwischen klar sein: für jene Lücke, die entsteht, wenn eine Gesellschaft ihr Personal nicht mehr eindeutig zuordnen kann und man sich dann darauf einen Reim machen muss" (Nassehi 2019, Pos. 2679); Christian Schüle, die semantische Offenheit nutzend, definiert: "Heimat ist die Gretchenfrage der Epoche" (Schüle 2018, 13).

,Heimat' wird, so meinen wir, schon in dieser konstitutiven Unschärfe als prekär markiert. Die Unterscheidung von "konkreter Heimat" und "Heimatbegriff" unterläuft alle Vorhaben, den "Konstruktionscharakter der Heimat" (Nell 2019, 119) auszustellen, weil hinter dem "Begriff" immer eine echte, wirkliche und vor allem nicht zu leugnende ,Heimat' stehen soll. ,Heimat' erhält so den Status einer intuitiven Evidenz, die diskursstrategisch behauptet und zugleich der Diskussion entzogen wird. Wer den Bezug zur ,Heimat' verloren hat oder sie als legitimatorischen Bezugspunkt für die Durchsetzung von Normvorstellungen in Frage stellt, diskreditiert sich in den Augen der ,Heimat'-Schützer genau damit als ein "anyw-

3 Edoardo Costadura, Klaus Ries und Christiane Wiesenfeld verstehen ,Heimat' davon ausgehend als Instrument, um die "ureigene Angst, der Natur ausgesetzt zu sein" zu bewältigen (dies. 2019, 19). here", der die eigene Wurzellosigkeit unrechtmäBigerweise zum Programm erhebt. ${ }^{4}$

Wir haben uns entschieden ,Heimat' grundsätzlich in einfache Anführungsstrichen zu setzen. Dass die Lesbarkeit dieses Textes dadurch nicht erleichtert wird, nehmen wir nicht nur in Kauf; vielmehr ist es unser Ziel, selbstverständliche Lesarten von ,Heimat' zu stören. Nicht etwa, um zu behaupten, dass der Ausdruck, die Idee und die Kulturgeschichte von ,Heimat' nicht wirksam sind und waren (und sein werden), sondern, um die Gefahr einer Essentialisierung von ,Heimat' programmatisch zu markieren. Das heißt nicht, dass ,Heimat' für uns persönlich keine Rolle spielt oder dass wir jemandem den Gebrauch des Wortes verbieten wollen (oder glauben, das tun zu können), aber die störenden Zeichen stellen aus, dass ,Heimat' prekär ist, insofern sie zwar Wirklichkeiten herstellen kann, aber nicht wirklich ist.

Dass der Ausdruck ambivalent ist, dass es so viele Definitionen für ,Heimat' gibt, wie Texte darüber (und mehr), mag daran liegen, dass ,Heimat' tatsächlich nicht als Begriff, sondern als "Assoziationsgenerator" verstanden werden sollte (Gebhard/Geisler/Schröter 2007, 9). Mehr noch, so unsere These, ,Heimat' ist die konzentrierte Form eines Entstörungsversuchs. ,Heimat' ist nichts, 'Heimat' tut etwas. Sie setzt jedoch nicht, etwas' an eine ,Leerstelle', ein unbefriedigtes Verlangen oder gar ein "ursprüngliches und natürliches Bedürfnis" (Costadura/Ries/Wiesenfeld 2019, 18), sondern stellt sicher, dass die für Gesellschaft konstitutiven Friktionen und Brüche latent bleiben. ,Heimat' ist also ein affektorganisierendes Verfahren der Vereinfachung, dessen Ergebnis gerade nicht Sicherheit, sondern - kontrastiv zu allen affirmativen Zuschreibungen - Prekarität ist. Was es mit dieser - zunächst kontraintuitiven - Behauptung auf sich hat, führt das vorliegende Sonderheft der Kulturwissenschaftlichen Zeitschrift vor: Es versammelt Perspektiven auf ,Heimat' als ge- und verbrauchtes Konzept, das es in seiner Prekarität zu rekonstruieren gilt. Damit schließen wir uns der "Distanznahme" an, die Armin Nassehi im Editorial des Kursbuch (2019) vorschlägt. Auch uns interessiert nicht, was ,Heimat' ist, sondern, was die Verfahren, Funktionen

$4 \mathrm{Vgl}$. zu der von Alexander Gauland im rechtskonservativen Diskurs populär gemachten Gegenüberstellung von "Somewheres" und „Anywheres", Mrozek 2019. 
und Ansprüche des Ausdrucks sind, welche Kontexte und Umwelten ihre Artikulation herstellt und/oder stört und, vor allem, wie und warum sie immer wieder eine solche Diskursgewalt entfalten kann.

Die Aufsätze dieses Sonderhefts entstammen einer Reihe von Workshops, die im Jahr 2018 an der Technischen Universität Dresden das Verhältnis von Heimat, Natur und Ökologie zum Thema hatten. Heimat, so war es geplant, sollte als ein besonders anschlussfähiger Aspekt des Projekts "Prekäre Natur" (Nitzke 2018a) zum Untersuchungsgegenstand für eine ökologisch orientierte Re-Lektüre von Texten ab 1840 werden. Weil aber die Institutionalisierung durch die Neugründung des deutschen Innen- als Heimatministerium 5 und die Diskussion von ,Heimat' quer durch alle Gesellschaftsbereiche in der Zwischenzeit ebenso rasant Fahrt aufnahm, wie der Widerstand gegenüber einer allzu zögerlichen Umwelt- und Klimapolitik, gewannen die Diskussionen über die ökologischen und globalen Dimensionen von ,Heimat' eine durchaus unerwartete Brisanz. Was die Beiträge und Beiträger*innen des Sonderhefts, uns eingeschlossen, verbindet, ist einerseits also das Nachdenken über Konzepte der Zugehörigkeit in Literatur, Theorie und populären Diskursen und andererseits die Skepsis gegenüber essentialisierenden Konzepten, die Kultur und Natur in reaktionärer Weise gleichsetzen und so Verhältnisse unhinterfragbar machen. Als "Distanznahme" streitet auch diese Sammlung von Texten nicht "für oder gegen einen Heimatbegriff" (Nassehi 2019, Pos. 39), sie gibt sich aber auch nicht mit einer Beobachtungsposition zufrieden: Vielmehr ist es das Ziel dieses Sonderhefts, ,Heimat' nicht nur als Komplexitätsreduktionswunderwaffe zu identifizieren, sondern die mit ihrer Indienstnahme produzierten Narrative und Mythen zu stören. Am Anfang steht dabei die Beobachtung, dass ,Heimat' nie stabil, sicher und Garantin von Geborgenheit gewesen ist. Das Wissen um die Prekarität von ,Heimat' scheint in den melancholisch-nostalgischen oder konservativ-restaurativen Varianten der Moderne verloren gegangen, oder von ihren Akteur*innen verdrängt worden zu sein. Das klingt zunächst danach, als ob es nur reaktiviert werden müsse, um die ,Heimat' von ihrer diskursiven Toxizität zu befreien. Das wiedergewonnene Wissen um den "Konstruktions- und Vorläufigkeitscharakter der Heimat" könne dann, so etwa Werner Nell, „eine davon ausgehende Einladung oder Nötigung zur Gewaltanwendung innerhalb von Heimatkonzepten" mäßigen (Nell 2019, 119). Aber lässt sich ,Heimat' so selbst stabilisieren? Wird der Ausdruck damit nicht endgültig unbrauchbar, weil seine Prekarität dann nicht mehr zu leugnen ist? ,Wohnen' und ,leben' lässt sich in einer solch fragilen, vorläufigen Heimat wohl nicht - oder ist es das, was ,wir' ohnehin schon die ganze Zeit tun?

Werner Nells Überlegungen zu Heimatdiskursen und Gewalt kommen zum wahrscheinlich vernünftigsten und vielleicht auch frustrierendsten Schluss innerhalb der aktuellen Ausprägung der Heimatdebatte. Anders als in vielen der seine Überlegungen umgebenden Texte, verspricht Nell weder die Rehabilitation, noch die progressive Aneignung des belasteten Konzepts oder auch nur des Wortes. Vielmehr verlangt er von einem "zeitgemäße[n] Heimatbegriff”, dass seine "implizierte Gewalthaltigkeit [...] in Rechnung gestellt und öffentlich reflektiert werden [muss]" (120). Das ist ein starkes Plädoyer gegen essentialisierende Indienstnahmen von ,Heimat'. Es beharrt darauf, dass viele der Wiederbelebungsversuche eines positiven, d.h. nicht wenigstens potenziell gewalttätigen oder menschenverachtenden, Anwendungsmodus von ,Heimat' zum Scheitern verurteilt sind, weil sie nicht nur an dem Ausdruck, sondern auch am Konzept selbst festhalten. Frustrierend ist Nells Schluss, weil selten so deutlich wird wie hier, dass man ,Heimat', selbst, wenn man es wollte, auf absehbare Zeit nicht loswird. Das gilt für dem Klischee-Diskurs der wirklichen, echten, ach so konkreten ,Heimat' ohnehin, weil sie dort ja immer erst aus der Ferne oder im Moment ihres Verlusts als solche erkannt wird.

Unsere Perspektive einer "Prekären Heimat" meint diese vielleicht empfundene und gegebenenfalls auch nachvollziehbare, materielle Gefährdung, den lebensweltlichen Verlust sowie das damit verbundene nostalgische Sehnen durchaus mit. Im Fokus steht jedoch die rhetorische, narrative und epistemologische Prekarität eines Konzepts, das sich vor allem durch die mit inm verschalteten Deutungsimperative auszeichnet. All die optimistischen Versuche, "Aufklärungsarbeit über ein deutsches Schlüsselwort zu leisten" (Scharnowski 2019a, 14), ,Heimat' einer pluralistischen Neukonfiguration zu unterziehen oder auch das "Versprechen, eine Resonanzbeziehung 
zur Welt einzugehen" (Rosa 2019, 153), doch noch einzulösen, lassen sich aus dieser Perspektive als zwar wohlmeinende, letztlich aber resignative Ansätze begreifen, an ,Heimat' und ihrem Resonanz- und Stabilitätsversprechen doch noch festzuhalten. Da das Scheitern oft nicht reflektiert wird, und so droht, den rhetorischen und ideologischen Ausgangspunkt einer (erneuten) Restauration zu bilden, unternimmt dieses Heft den Versucht, Resignation und Resonanz gleichermaBen zu stören. Dementsprechend verstehen wir unseren Text nicht als erneuten Versuch, einen Forschungsstand zu synthetisieren, sondern als Sabotageakt an der Evidenzmaschine ,Heimat'.

\section{Gegen ,Heimat', oder: was nicht „Heimat' ist}

Bei aller rhetorischen Dringlichkeit scheint es doch so, als ob der Streit um einen vermeintlichen "Kampfbegriff" (Scharnowski 2019a, 9) trotz aller Beteuerungen der politischen Relevanz meistens ein rein akademisches Vergnügen ist. Weder in den Supermärkten, noch in städtischen und regionalen Marketingkampagnen, Reiseprospekten oder in den Wahlkämpfen diverser Parteien lässt sich ein Nachlassen der Heimatbegeisterung feststellen. Ganz im Gegenteil scheinen die diffusen Konturen des gleichermaßen unterbestimmten wie überdeterminierten Ausdrucks sicherzustellen, dass jedes Thema und Produkt, jedes Versprechen als ,Heimat'-Kompositum gerahmt werden kann. Das mag daran liegen, dass, Heimat' schon alles zu sagen scheint und nur noch mit den Spezifika der eigenen Erfahrung geschmückt werden muss, um persönlich zu werden. In den Händen von Akademiker*innen hat ,Heimat' also nichts verloren dafür spricht schon die Menge an Anführungszeichen, die wir in diesem Text bereits benutzt haben. Sind aber nicht gerade Kulturwissenschaftler*innen allzu begeistert von nomadischen Konzepten, pluralen und pluralistischen Verhältnissen von Mensch, Raum und Natur und wären ganz einverstanden damit, den ,Ort' und die ,natürliche Zugehörigkeit' gleich auf die Müllhalde untauglicher Kategorien und Konzepte zu werfen? Wie könnte auch, so eine beliebte küchenpsychologische Argumentationsfigur, das vielgerühmte ,Heimatgefühl' bei Leuten entstehen, die qua Beruf ständig gezwungen sind, die Zelte abzubrechen und die oft nicht länger als ein paar Jahre in die Zukunft planen können. Anstatt aber ihr Sehnen nach Sicherheit zuzugeben, diskreditieren diese Akademiker*innen - auch wir - ,Heimat' wo sie können. Was sie nicht haben können, steht demnach auch niemandem sonst zu.

Vielleicht ist es genau diese Karikatur des (Selbst-)Bildes heimtatloser und heimathassender Akademiker*innen, die die neuerliche Entschlossenheit anfacht, mit der manche Literatur- und Kulturwissenschaftler*innen ihr kulturelles Kapital einsetzen, um an ,Heimat' festzuhalten. Die sozioökonomische Prekarität und Ortlosigkeit des akademischen Betriebs auf der einen und der unbedingte Wille eine oft gleichermaßen gedankenlose wie bedenkliche gesellschaftliche Begriffssetzung zu kommentieren auf der anderen Seite, führen zu einem Wettstreit, um ,Heimat', der nicht zu gewinnen ist. Denn, wer ließe sich ernsthaft aus Richtung der Universität sagen, wo ihr oder sein ,Heimatbegriff der Umdeutung bedarf? Weil ,man' ja ohnehin intuitiv weiß, was ,Heimat' ist - immer und vor allem für einen selbst - sind alle Kulturgeschichten höchstens schmückendes Beiwerk. Lassen sich die vielen mehr oder weniger institutionell verankerten ,Heimat'-Erkundungen der letzten Jahre also als Versuch lesen, sich selbst eine ,Heimat' zu erschreiben - und sei es nur in der Theorie? Sind sie Ausdruck einer Sehnsucht nach Ortsgebundenheit aus ausgerechnet der Richtung, die Mobilität, Flexibilität und Kosmopolitismus so ausstellt? Jedenfalls scheinen die neoliberalen Fliehkräfte und die Enthausungstendenzen einer sich immer weiter globalisierenden Welt ${ }^{5}$ den neuen ,Heimat'Theoretiker*innen (und auch der "Neuen" Rechten) in die Karten zu spielen. Die akademischen "Heimatskeptiker" (Scharnowski 2019a, 15) ${ }^{6}$, so

5 Vgl. Bauman 2013; s. auch Stephanie Heimgartners Beitrag „Flüssige Identitäten. Identitätspolitik in den Texten der afrikanischen Diaspora".

6 Es ist bezeichnend, dass Scharnowski mit dem Terminus "Heimatskeptiker" einen Ausdruck wählt, der metaphorisch und semantisch an den Kampfbegriff "Klimaskeptiker" anschließt. Sie vollführt damit, ob beabsichtigt oder nicht, eine rhetorische Volte, die beide "-skeptiker" als Leugner eindeutiger, d.h. hier: wissenschaftlich belegter Wirklichkeiten ausweist. Gleichzeitig appropriiert dieser Terminus einen Diskurs, dessen "grüne" Markierung ihr selbst als Sprecherin Legitimität für ihre "konservativen" Schlüsse verleiht. Im Grunde handelt es sich dabei um eine Art "Greenwashing" einer ,Heimat'-Rehabilitation. Scharnow- 
das suggestive Argument, bedienen sich demnach einer affirmativen Selbsttäuschung: So würden die Kritiker*innen des, Heimat'-Begriffs eigentlich selbst nichts mehr wollen, als endlich ihre Skepsis dranzugeben und sich dem Sog und der Sicherheit der ,natürlichen Heimat' zu ergeben. Da dies aber aus Distinktionsgründen und den Effekten eines diskursiven Mainstreamings unmöglich sei, komme es zu einem Überbietungswettbewerb der Distanzierung und Dekonstruktion.

Interessant am ,Heimat'-Diskurs erscheint uns, dass die Debatte oftmals in Registern des Emotionalen geführt wird. Dann ist schnell von Kompensation, Verdrängung etc. die Rede. Uns scheint es demgegenüber produktiver, eine funktionale Perspektive auf die Rede über die, Heimat' zu richten. Nicht was letztgültig ,Heimat' ist, interessiert dann, sondern wozu der Bezug auf ,Heimat' genutzt werden kann. ,Heimat', so unsere These, fungiert, insbesondere dort, wo sie mit dem weiteren Letztbegründungsbegriff ,Natur' verschaltet wird, als ein paradoxes Instrument narrativer Welterzeugung. Paradox insofern, als sie sich immer schon und unauflöslich nur im Modus der Störung thematisieren lässt. ${ }^{7}$,Heimat' erzeugt Evidenz ohne Transparenz: Einerseits weiß man vermeintlich intuitiv, was ,Heimat' ist. Man fühlt, riecht und schmeckt sie und es ist sofort klar, wer sie teilt. Andererseits kann sie erst dann zum explizierbaren Thema werden, wenn sie den Status selbstverständlicher Zuhandenheit verloren hat. Erst dann also, wenn sie durch einen Einbruch des Anderen bedroht erscheint, wenn ihre Kontinuität zum Problem geworden ist. Spätestens hier - dies wäre einer der Hauptattraktoren des deutschen Heimatfilms in den 1950er Jahren

skis selbsterklärtes Aufklärungsprojekt wird zusätzlich fragwürdig, wenn sie in der medialen Begleitung ihres Buches mit rhetorischen Kurzschlüssen wie dem folgenden argumentiert: "Also was mir wirklich ein wichtiges Anliegen ist, dieser Gemeinplatz, dass das Wort Heimat verbrannt sei, weil es Nationalsozialisten propagandistisch genutzt hätten, halte ich für verfehlt aus zwei Gründen. Der erste Grund ist, man muss ja zunächst mal Propaganda und Politik voneinander trennen, und wenn wir schon uns entnazifizieren wollen, wofür ich sehr bin, dann würde ich sagen, schaffen wir doch als erstes mal die Autobahnen ab. [...] warum versteifen wir uns so auf die Heimat, warum ist es die Heimat, die so als kontaminiert gesehen wird, wo es ja noch ganz andere Aspekte gäbe." (Scharnowski 2019b). 7 Zur narrativen Funktion von Störung vgl. Koch/Nanz/ Pause 2016.
- trifft in einem Szenario von De- und Renormalisierung, Irritation und Entstörung, ,Heimat' auf ,Natur'. Beide produzieren vor dem Horizont eines drohenden Entzugs Evidenz und Zugehörigkeit und beide stehen, so formuliert es W.G. Sebald, "in einem reziproken Verhältnis zu dem, worauf [sie] sich bezieh[en]. Je mehr von der Heimat die Rede ist, desto weniger gibt es sie." $(1995,12)$

In dieser Hinsicht scheint Susanne Scharnowskis Diagnose zuzutreffen, dass es "nicht etwa ein Zuviel an Heimat gibt, sondern eher einen Mangel" (Scharnowski 2019a, 16). Sie bringt damit eine Haltung zum Ausdruck, die die Privilegiertheit der eigenen Position verkennt. Denn die Vorstellung, man müsse bloß er- und anerkennen, dass ,Heimat' - sobald man sie endlich nicht mehr ständig als „verbrannt" abtue - für alle da sei, steht nur denen offen, deren Anspruch auf ,Heimat' aus der Gewissheit resultiert, Anspruch auf Zugehörigkeit und Heimat zu haben. Nur von einem solch unhinterfragtem Besitzstandsdenken her ist es möglich, Kritik an ,Heimat' als ideologisch verbrämte Angriffe von links zu diffamieren.

Konkrete Schilderungen davon, dass ,Heimat' keinesfalls ein "Phantomschmerz" (Schüle), sondern "ein Albtraum" (Aydemir/Yaghoobifarah 2019) ist, sind indes kaum zu übersehen. Daniel Schreibers autobiographischer Essay Zuhause. Die Suche nach dem Ort an dem wir leben wollen (2017) gehört neben der von Fatma Aydemir und Hengameh Yaghoobifarah herausgegebenen Anthologie Eure Heimat ist unser Albtraum (2019) und Max Czolleks Polemik Desintegriert Euch (2018) wohl zu den sichtbarsten Gegenentwürfen zu den scheinbar allgegenwärtigen Wiederbelebungsversuchen. Sichtbarkeit und die prejorisierende Markierung von Non-Konformismus ist diesen , Heimat'-kritischen Interventionen als zentrales Thema gemeinsam. Klar wird bei der Lektüre, ,Heimat' ist nicht etwa ein verlorener Ort, der bloß erinnert werden kann. ,Heimat' ist vor allem ein diskursives und soziales Invektivgeschehen $^{8}$, das anhand der gewaltsamen (Durch-) Setzung einer ,natürlichen' Dominanzkultur unter der Forderung nach Integration Abweichungen sanktioniert. Um das "ursprüngliche Bedürfnis nach Heimat" (Costadura/Ries/Wiesenfeldt $2019,18)$ erfüllen zu können ist es diesen Tex-

8 Vgl. hierzu Konzeptgruppe „Invektivität”, Ellerbrock et al. 2017. 
ten zufolge nötig, einer Norm zu genügen, die der eigenen individuellen Lebenswirklichkeit die Geltung abspricht. Es ist nach der Lektüre dieser Texte nicht mehr so einfach, an die Möglichkeit einer pluralistischen Neubestimmung der Zukunft des multi-ethnischen Zusammenlebens zu glauben. Zwar mag ,Heimat' das Symptom eines Mangels sein, aber die Restituierung der mit ihr verbundenen normativen Ansprüche kann die Leerstelle, auf die sie reagiert, offenbar nur füllen, indem sie eine Form exklusiver Solidarität imaginiert, die für eine begrenzte Zahl an Menschen Vertrautheit und Geborgenheit gewährleistet, für ,den Rest' aber eine unüberwindbare Barriere errichtet, hinter der sie als verworfenes Leben ihr Dasein in der totalen Freisetzung fristen müssen. ${ }^{9}$, Heimat' stabilisiert sich also, indem sie andere ausschließt und diesen Ausschluss durch die Behauptung und Durchsetzung einer, natürlichen' (mindestens aber ,traditionellen') Zugehörigkeit legitimiert. ${ }^{10}$ Das heißt, sie definiert sich, vor allem über das, was sie nicht ist. Das Problem ist demnach nicht der "Mangel an Heimat", sondern der inhärente Mangel, den ,Heimat' selbst erzeugt - ein Mangel an Vielfalt und Offenheit, der Sicherheit durch den hochinvektiven - mindestens symbolischen, oftmals aber auf manifest praktischen - Ausschluss von Abweichung herstellt. Dass dieser Mangel konstitutiv ist, und nicht destruktiv für die Haltbarkeit der Idee, scheint weder bei Schüle, noch bei Scharnowski eine Rolle zu spielen. Insbesondere letztere scheint die, unheimlichen' Eigenschaften von ,Heimat' als bloßen Effekt polarisierter Pro- und Anti-Heimat-Diskurse zu verstehen. Dabei ist es gerade die Unheimlichkeit der ,Heimat', die sie zu einem Motor des literarischen wie (auto-)biographischen Erzählens macht. Egal wie sicher man sich ihr zu sein scheint, egal, wie weit entfernt von der ,Heimat' die Erzählenden sich befinden, ob "Phantomschmerz" (Schüle) oder "schönste Utopie" (Menasse 2014), die Anziehungskraft von ,Heimat' speist sich in nicht geringem Maße daraus, dass sie sich entzieht, dass sie gerade nicht stabil und verlässlich ist, sondern unter oder hinter dem Sichtbaren etwas Unerwünschtes, Beängstigendes lauert, das Zugehörigkeit bestimmt.

9 Vgl. Bauman 2005; Brown 2010.

10 Vgl. Teil zu „Eigenart” bei Günzel 2014.

\section{Heimatinstinkte und Verwurzelungsversuche}

Das Konzept der "unheimlichen Heimat" stammt von dem Literaten und Literaturwissenschaftler W.G. Sebald, der es zum gemeinsamen Bezugspunkt einer Reihe von Essays über österreichische Literatur macht. ,Heimat' ist für inn kein Ort, sondern der kleinste gemeinsame Nenner der österreichischen Literaturgeschichte: „Es ließe sich die Auffassung vertreten, daß die Beschäftigung mit der Heimat über alle historischen Einbrüche hinweg geradezu eine der charakteristischen Konstanten der ansonsten schwer definierbaren österreichischen Literatur ausmacht [...]" (Sebald 1995, 14). Zu diesen "historischen Einbrüchen" zählt Sebald sowohl die „Entwicklung, die Österreich von dem weit ausgedehnten Habsburger-Imperium zur diminutiven Alpenrepublik" durchlaufen hat, als auch den "Anschluß an das unselige Großdeutschland" (Sebald 1995, 14). ,Heimat' wird hier zum impliziten, imaginierten Kern eines gemeinsamen Bezugsraums, der keine stabilen (Außen-)Grenzen hat. Sie wird also in der Tat zur "Ersatzhandlung" (Nassehi), die Zusammenhang stiftet, wo Gemeinsamkeit zwar ersehnt wird, aber weder eine materielle noch eine historisch stabile Grundlage hat. Der "Heimatbegriff", so Sebald, entspringt im 19. und 20. Jahrhundert der Erfahrung des unhintergehbaren "Heimatverlusts" durch Vertreibung und Auswanderungsdruck:

Doch zeigt es sich auch zu Hause, wie in der Prosa
Stifters allenthalben nachzuweisen wäre, daß das Ver-
hältnis der Menschen zu ihrer angestammten Heimat
gebrochen ist, von dem Augenblicke an, da diese ein
literarisches Thema wird. [...] Die Stifter als Heimat-
schriftsteller reklamierten, übersahen, wie unheim-
lich ihm die Heimat geworden war. Überall herrscht
die größte Kälte, in den Verhältnissen zwischen den
Menschen sowohl als in der in ihrem Bewußtsein auf
einmal als, das andere' aufgegangenen Natur. (Sebald
1995, 15)

Stifters exzessive Aufmerksamkeit für die nichtmenschliche Umwelt entspringt insofern einer Verunsicherung bzw. einer wachsenden Skepsis gegenüber der ,Natürlichkeit' von ,Heimat'. Selbst dort, wo ,Natur' in allen Einzelheiten bekannt ist, bewegen Menschen sich in ihr wie Fremde. Das heißt nicht, dass sie sich in ihr nicht zurecht- 
finden könnten, wohl aber dass sie nicht mehr unproblematisch ein Teil von ihr sein können, weil der "anhebende Hochkapitalismus [...] wie die Geschichte einer zweiten Vertreibung" (Sebald 1995, 15) daherkommt. ${ }^{11}$ Sebald nimmt diese Beobachtung partiell aber wieder zurück, wenn er feststellt, dass sich die Auseinandersetzung mit der "unheimlichen Heimat" in der jüngeren österreichischen Literatur auf die "natürliche Heimat des Menschen" (Sebald 1995, 16) erstrecke. Nicht mehr nur die unhinterfragbar ,natürliche' Zugehörigkeit zu einer sozialen oder nationalen Gruppe - zu anderen Menschen also -, sondern auch das Verhältnis zur nicht-menschlichen Natur steht einmal mehr in Frage. Allerdings verschiebt sich der Fokus: War es bei Stifter vor allem der Prozess der Vergemeinschaftung, der seine Selbstverständlichkeit verliert, geht es nunmehr um das Fortbestehen der "natürlichen Heimat" selbst. Die "angst- und ahnungsvolle Aufzeichnung der Veränderung des Lichts, der Landschaft und des Wetters" (Sebald 1995, 16) deutet darauf hin, dass die unheimliche Qualität von ,Heimat' sich mit einem Schlag auf die gesamte nicht-menschliche Umwelt, den ganzen Planeten ausweiten kann. ${ }^{12}$ Unheimlich wird ,(natürliche) Heimat' in diesem Sinne dann, wenn ins Bewusstsein drängt, was sonst latent bleibt: dass sie sich verändert, dass ihre Zukunft nicht abzusehen ist, kurz, dass sie prekär ist.

Es ist vor diesem Hintergrund kein Zufall, dass im Zusammenhang mit, Heimat' ökologische Metaphern eine so starke Rolle spielen. Dabei sind es vor allem die, die eine vermeintlich feste, unverbrüchliche Verbindung anzeigen, die das semantische Repertoire des ,Heimat'-Diskurses dominieren: bei den Körpermetaphern ist es weniger der Atem- als der Blutkreislauf (mit dem Boden), der ,natürliche' Zugehörigkeit evident macht. Dementsprechend versetzen sich Menschen auch weniger in auto-mobile Tiere hinein, um sich narrativ selbst zu lokalisieren, denn in Pflanzen. Weil diese in der Erde wurzeln, werden sie zu einer Identifikationsfigur. Im Begehren sich - wie Bäume und mit Bäumen - an einen Ort zu binden und die Bäume zu Zeugen (und Zeugnissen) der eigenen Geschichte

11 Vgl. hierzu Nicolas Pethes Beitrag: „Der Heimgarten. Zur Konstruktion einer Semantik in Zeitschriften/Literatur des 19. Jahrhunderts".

12 Vgl. zu Sebald und „natürlicher Heimat”: Nitzke 2018b. zu machen, wird ,Heimat' zum prekären Naturverhältnis. Besonders hier wird die Verbindung von "unheimlicher" und "natürlicher" ,Heimat' evident, denn der Versuch, ,Heimat' herzustellen, indem man sich an einem Ort verwurzelt, ist weit davon entfernt, ein Neubeginn zu sein. Vielmehr zeigt sich gerade hier, wieviel Gewalt nötig ist, um die eigene Verbundenheit mit ,dem Boden' zu plausibilisieren. Auch hier ist also, was zunächst wie ein ,natürliches', als a priori legitimes Bedürfnis erscheint, Ausdruck einer problematischen Leugnung einer für die Modere konstitutiven Entfremdungserfahrung. ${ }^{13}$

Die damit angesprochene, latente Gewaltgrundierung der ,Heimat'-Emphase lässt sich exemplarisch auch an Bernd Heinrich Buch Heimatinstinkt (The Homing Instinct 2014; dt. 2016) beobachten: Diese autobiographisch gerahmte, populärwissenschaftliche Überblicksdarstellung eines deutschamerikanischen Biologen beschäftigt sich mit dem Heimfindevermögen verschiedener Tierarten. Dass Heinrich seine über Strecken deutlich konservativ gefasste Autobiographie als Rahmen für die Erkundungen nicht-menschlicher Verhaltensweisen nutzt, ist gleich im doppelten Sinne als Indiz einer Naturalisierungsstrategie signifikant: Zum einen macht er so die eigenen Lebensentscheidungen mit dem vollen Gewicht seines professionellen Wissens und der daran gebundenen Autorität unhinterfragbar. Zum anderen wird hier der Versuch erkennbar, (die eigene) Geschichte unter der Perspektive eines ,biologischen' Gefühls als Teil einer planetaren, ökologischen Gemeinschaft, also in Kontinuität zu anderen Lebewesen zu erzählen. Diese Geschichte ist eine des Pflanzens: Die selbst gepflanzte Kastanienallee, welche den Vater des Erzählers an dessen, alte Heimat' in Europa erinnern, sowie ein alter Apfelbaum, der seine Geschichte, wenn auch widerwillig und um den Preis seiner Zerstörung, als "geschlossene lange Kette [preisgibt], die bis zu den ersten Siedlern dieses Gebiets, zu den Männern und Frauen, die

13 Wie diese Kompensation in politische Agitation umschlagen kann, hat Erhard Schütz anhand des NS-Propagandafilms "Ewiger Wald" (1936) gezeigt, in dem zu Bildern wogender Bäume eine Erzählstimme verkündet: „Aus dem Wald kommen wir / wie der Wald leben wir / aus dem Wald formen wir / Heimat und Raum." Schütz 2019, S. 85-152; Vgl. zur problematischen Verquickung von 'Heimat' und 'Umwelt' auch Scharnowski 2016. 
die amerikanische Geschichte gestalten hatten," zurückreicht (235) - es sind die fest verwurzelten Bäume, die den "Heimatinstinkt" des lange an der Universität von Vermont lehrenden Erzählers aktivieren und dessen Heimatsehnsucht zu befriedigen vermögen:

Ein Heim zu gründen heißt, an einem bestimmten Ort ,Wurzeln zu schlagen', beständig genug zu sein, um in Zeiträumen zu denken und zu wirken, die Lebensspannen umfassen, und physische Spuren zu hinterlassen, etwa durch die Anordnung von Feldsteinen oder das Einpflanzen von Apfelbäumen. (Heinrich 2016, 224)

Das bedeutet allerdings auch, dass diejenigen Bäume, die das ruinierte Haus auf Heinrichs Grundstück überwuchert haben, weichen müssen, auch wenn sie nicht weniger fest verwurzelt sind als ihre von Menschen gepflanzten Artgenossen. Heinrichs Narrativ knüpft explizit an das der amerikanischen Homestead-Bewegung, sowie implizit an die damit verbundenen Natur- und WildnisNarrative an. ${ }^{14}$ Damit positioniert sich sein Text in seinem Verwurzelungsethos zwischen Autobiographie und (Populär-)Wissenschaft - womit er ganz im Rahmen des Nature Writings bleibt - lehnt aber die genretypische Gegenüberstellung von Ich und Natur, die erst in eine (langsame und tentative) Annäherung münden soll, grundsätzlich ab. ${ }^{15}$ Die Beobachtung der Natur dient bei Heinrich einzig ihrer sinnvollen und nachhaltigen Nutzbarmachung. In Erfüllung eines dem schreibenden Ich als exemplarischem Individuum der Gattung Mensch eingeschriebenen "Heimatinstinkts/homing instinct" wird "Natur' zu Herausforderung und Ressource anthropogener Gestaltung. Heinrich produziert also auf allen Ebenen des Textes Ordnung. Diese Ordnungen legen sich über offenbar komplexe und z.T. komplizierte Beziehungen, die durch entschiedene Eingriffe übersichtlich gemacht werden: Beziehungen des Ichs zu Natur und Kultur - gepflanzte Natur hier, wilde Natur dort; verdorbene urbane Welt möglichst ganz woanders -; zu anderen Menschen - eingangs Kommentare über Schwestern und Mutter, die Beziehungen offenbar haben, eingehen lassen', weil sie nicht in Heinrich Memoiren über seinen Vater auftauchen; sowie die beobachtete und behauptete Ähnlichkeit des Verhal-

14 Vgl. Cronon 1996.

$15 \mathrm{Vgl}$. Fischer 2019. tens von Menschen und Tieren, die schon in der Kapitelordnung des Buchs plausibilisiert und im Text durchgehend narrativ in Beziehung zueinander gesetzt werden. Die ordnende Arbeit, welche Heimatinstinkt kennzeichnet, ist eine in erster Linie narrative. Gerade, weil Heinrich Erzähler-Ich immer wieder betont, dass die Verknüpfungen von Autobiographie und ,Naturkunde' nicht fiktional sind, wird darin die Absicht deutlich, einen Eindruck narrativer Organizität zu erzeugen. Verwurzelung, Vertrautheit und Natur werden in der naturalisierten ,Heimat' verschaltet. Interessen und Ordnungsprinzipien aus den Baum-Kapiteln sind als poetische Verfahren auch in der Logik des gesamten Textes sichtbar. So wird trotz des impliziten Anschlusses an Ideen einer ,echten' Natur (des Menschen) keine Wildnisromantik betrieben. Im Gegenteil, das engste Umfeld eines sich verwurzelnden, verheimatenden Menschen bietet gerade keinen Raum für Wildwuchs, denn es ist die Ordnung und Gestaltung von Natur nach menschlicher Vorstellung, die Heimat als (Natur-)Raum des Menschen überhaupt erst als solche sichtbar macht. Heinrichs Text lässt sich in diesem Sinne als Abwehrunternehmung lesen, die mit Macht versucht, das Unheimliche der ,Heimat' auszuschließen. Mit Macht, fast schon mit Gewalt, wird alles, was sich jenseits des Willens desjenigen, der einen Ort zu , seiner Heimat' machen will, bewegt, zu ihrem Teil gemacht oder als ihr Anderes verworfen. Was sich diesem Projekt nicht unterordnet, verschwindet. Doch auch dort, wo, geradezu ausgestellt wird, dass ,Heimat' gemacht, nicht (wild) gewachsen ist, bleiben Brüche und Reste sichtbar, die bei genauerem Hinsehen ein Insistieren von Unheimlichkeit und Prekarität anzeigen.

\section{Heimatforschungsforschung}

,Heimat' zu machen ist, das zeigt Heinrichs Beispiel ${ }^{16}$, ein Privileg. Das gilt vor allem für diejenigen, die für sich reklamieren, "Heimat' zu "finden" oder einfach zu "haben". "Heimat' als Telos der eigenen Biographie verhindert ebenjene Offenheit, die nötig ist, um Ansprüche an

16 Zur problematischen Verherrlichung verwandter Versionen dieses „Ein-Mann-im-Wald-Narrativs" vgl. Nitzke 2020. 
Pluralität, Diversität und Freiheit zu realisieren, wie sie Aydemir, Yaghoobifarah, Czollek u.a. formulieren. Werden sie, wie in Stanišićs Herkunft, konsequent in der Form umgesetzt, wird ,Heimat' als letztbegründender Bezugspunkt buchstäblich verspielt. Entsprechend suspekt sind die Versuche, ,Heimat' als Konzept zu rehabilitieren, umzudeuten und wieder, salonfähig' zu machen, denn sie laufen Gefahr, die spielerische Offenheit von ,Heimat' im Plural - von vielfältiger Herkunft als Ausgangspunkt, nicht als Markierung und Telos unhinterfragbarer Zugehörigkeit; von Gemeinschaft als Option, nicht als Schicksal - zugunsten einer prekären Pseudo-Stabilität zu zerstören. Die Beiträge, die dieses Sonderheft versammelt, werden selbst zu Störfällen ,natürlicher' Zugehörigkeit, insofern sie den Blick auf die Produktion und Konstruktion von ,prekärer Heimat' lenken. Sie sind in dieser Hinsicht keine Beiträge zur ,Heimat'-Forschung, sondern betreiben eine Heimatforschungsforschung, die ihre eigene Beobachter*innenposition immer mit in den Blick nimmt. Eine ,Heimat'-Forschung zweiter Ordnung ist notwendig komparatistisch und interdisziplinär angelegt und hat zum Ziel, Anschlusspunkte aufzuzeigen und damit Offenheit zu produzieren. Sie will Diskussion anregen und verweigert sich dezidiert Letztbegründungen und -bestimmungen. Die thematische Vielfalt ist also programmatisch: Sie verfolgt historische Linien der Plausibilisierung von ,Heimat', die zeitliche Ferne und Nähe ebenso instrumentalisieren, wie räumliche. Die versammelten Essays betrachten Akteure von Dinosauriern und Urmenschen bis zu Außerirdischen, Literaturen aus Deutschland, Österreich, Frankreich, Ghana, Zimbabwe, Senegal und den Vereinigten Staaten und Perspektiven, die einen einzelnen Acker oder die ganze Erde umfassen.

Nicolas Pethes untersucht ,Heimat' als Produkt der massenmedialen Kultur des 19. Jahrhunderts, des "Zeitalters der Zeitschrift". ,Heimat' ist nicht allein Produkt einer Verlusterfahrung, sondern Ergebnis journalistischer Strategien, deren Bedingungen und Konsequenzen Pethes in seinem Beitrag "Landschaft und Genealogie. Die Semantik von ,Heimat' in Zeitschriften/Literatur des späten 19. Jahrhunderts" im Vergleich von Adalbert Stifters Nachkommenschaft und der heute vergessenen Erzählung "Der Schütz' von der Pertisau" von Herman Schmid in Augenschein nimmt. Scheinbar geschlossene Heimgarten- bzw.
,Heimat'-Semantiken werden, das zeigt Pethes, in inrer Geschlossenheit erst in der Buchform produziert, während sie sich im Umfeld der Zeitschriftenpublikation vielfach gegenseitig unterlaufen. Pethes' Untersuchung der medialen Dimension der ,Heimat' ist für die gegenwärtige Diskurslage von enormer Bedeutung, weil sie zeigt, dass die Diversität von Mediatisierungen der ,Heimat' ihr nicht erst in der geschichtsvergessenen Gegenwart angehängt wird, sondern den Ausdruck und das Konzept zuallererst hervorgebracht hat und bis heute prekär stabilisiert.

In seinem Beitrag "Von Korallenatollen in deutschen Meeren und anderen Fundstücken aus dem steinernen Heimat-Archiv des Deutschen Reichs" widmet sich Patrick Stoffel der bisher wenig beachteten Frage nach der temporalen Ausdehnung von ,Heimat'. Er zeigt an populärwissenschaftlichen Schriften aus den Jahren 1898-1931 von Alfred Götze und Wilhelm Bölsche, dass im Deutschen Reich parallel zur räumlichen Expansion der Heimat auch eine zeitliche Expansion erfolgte, die über die Verortung der ,Heimat' in der, guten alten Zeit' weit hinausreichte und über die Ur- und Frühgeschichte bis tief in die Erdgeschichte ausgriff. Hier werden die Anschlussfähigkeit des Konzepts - und damit einer der Gründe für seinen enormen Erfolg - und seine Aporien aus einer Perspektive beleuchtet, die das Verhältnis von Mensch und Landschaft nicht als Ausgangspunkt, sondern als Ergebnis eines Erzählprozesses versteht, der gerade keine inhärenten Grenzen hat.

Peter Neumann schließt daran an, indem die ,Heimat'-Operation der scheinbar konkreten Ver-Ortung eines Menschen in der Landschaft untersucht. In seinem Beitrag "Der Deutsche in der Landschaft - Borchardt und Benjamin" geht Neumann von der methodologischen Kritik Benjamins an Borchardts Anthologie Der Deutsche in der Landschaft (1927/2018) aus um nachzuvollziehen, wie Borchardts Modell der, geistigen Zugehörigkeit' bei Benjamin unter den Vorzeichen des Faschismus in sich zusammenbricht und die Fiktion ,organisch gewachsener Kulturalität' sich als prekäres Zeugnis seines eigenen Untergangs erweist.

Natalie Moser fokussiert den Boden, von dem aus Zugehörigkeit konstruiert wird. In ihrem Beitrag "Heimat als vergiftetes und sich vergiftendes Ökosystem. Zur Überblendung von Heimat-, Öko- 
logie- und NS-Diskurs in Josef Winklers Laß dich heimgeigen, Vater, oder Den Tod ins Herz mir schreibe" untersucht sie die Praktiken des Verschweigens, Vertuschens und Versteckens sowie deren Scheitern. Darin legt der Josef Winklers Roman, so Moser, Kreisläufe als Thema, Motiv und poetisches Prinzip von offen und erlaubt die ,ökologische' Lektüre von ,Heimat' abseits allzu optimistischer umweltpolitischer Hoffnungen.

Anstelle einer lokalisierten oder regionalen Zugehörigkeit untersucht Uwe Lindemann in "Totale Entheimatung oder: Die Vertreibung des Menschen von der Erde. Über H.G. Wells' The War of the Worlds." den Planeten Erde als ,prekäre Heimat' des Menschen. Seine Lektüre des berühmten Science-Fiction-Romans legt offen, dass die Annahme der Erde als gleichsam ,ewiger Heimat' des Menschen eben nicht - wie bspw. in den von Patrick Stoffel untersuchten populärwissenschaftlichen Texten - die einzige Lesart des Verhältnisses ist. Gerade in Zukunftsfiktionen verschafft sich die Einsicht Raum, dass der Mensch bestenfalls eine Episode in der Erdgeschichte ist.

Daraus ergeben sich entscheidende Implikationen für den Diskurs der (politischen) Ökologie. In seinem Beitrag "Zur lyrischen Inszenierung ,natürlicher Heimat' - Der Blick auf den ,Heimatplaneten' in Durs Grünbeins Gedicht Tacchini (2014)" vollzieht Jonas Nesselhauf die Änderung der Blickrichtung nach, die Mitte des 20. Jahrhunderts ironischerweise mitten im "Space Race" zwischen den konkurrierenden Systemen die Frage nach den Grenzen des Imperialismus in den Vordergrund rückte. Während die imperiale Ausdehnung in der Imagination dieser Zeit keine Grenzen kannte, wurden die Limitationen der Erde als ,natürlicher Heimat' des Menschen scheinbar schlagartig sichtbar. Grünbein greift diesen Gestus in seinem Gedicht auf und stellt erneut die Frage nach der Berechtigung des Menschen, sich einen ganzen Planeten zur ,Heimat' zu machen.

Dass ,Heimat' in hohem Maße von den Wahrnehmungsgewohnheiten und -erwartungen abhängt, führt Elisabeth Heyne in ihrem Beitrag "Tropische Wahrnehmungshygiene. Zu natürlichen Sinneslehren im Amazonasgebiet (Claude Lévi-Strauss, Pierre Restany, Eduardo Kohn, Pola Oloixarac)" vor. Sie untersucht zwei Paradigmen einer amazonischen Wahrnehmungslehre, die den Regenwald als Hintergrund für die Erfahrung eines letzten Rests wilder Natur einerseits und als Ressource und Bezugspunkt einer neuen Sensibilität für die Verstrickungen zwischen Mensch, Umwelt, Tier, Pflanze und Technik im Anthropozän andererseits versteht. Anhand des Romans Constelaciones oscuras von 2015 (dt. Kryptozän, 2016) der argentinischen Autorin Pola Oloixarac bringt Heyne theoretische Konzepte aus ethnologischen Texten der Mitte des 20. Jahrhunderts und der unmittelbaren Gegenwart in Dialog. Auch hier - wie bei Wells - scheint die Zukunft nicht notwendig dem Menschen zu gehören.

Die Diskrepanzen und identitätspolitischen Konsequenzen, die sich aus einer solchen prekären Beziehung zu ,Heimat' als globaler und planetarer Größe ergeben, zeigt Stephanie Heimgartner in ihrem Beitrag „Flüssige Identitäten. Identitätspolitik in Texten der afrikanischen Diaspora" auf. Der Artikel diskutiert literarische Konzepte von Identitätsverflüssigung, Erfahrungen der Körperlosigkeit und Strategien des Namenswechsels an beispielhaften Texten von Léonora Miano, NoViolet Bulawayo und Fatou Diome und verdeutlicht, dass ein räumliches Konzept von ,Heimat' heute notwendigerweise nur noch als prekär darstellbar ist und andersartige Identitätskonzepte an seine Stelle treten müssen.

Sarah Heinz beobachtet in ihrem Beitrag "Emergent Homes. Negotiating Homing Desires through Doing Home in Yaa Gyasi's Homegoing" ähnliche Probleme und entwickelt angelehnt an Avtar Brahs Konzept des ,homing desire' (1996) und anhand der Lektüre von Yaa Gyasis Roman Homegoing (2016) einen Weg zur Konzeptualisierung von ,Heim/at' jenseits der potenziell problematischen Glorifizierung von Nomadismus und radikaler Heimatlosigkeit einerseits und Verwurzelung und auf Dauer gestellter Sesshaftigkeit andererseits. ,Heimat' realisiert sich hier als andauernde, nicht zu befriedigende Sehnsucht. Damit schlägt Heinz via Gyasi den Bogen zur aktuellen Debatte, die mit aller Macht versucht, die imaginäre Qualität' von ,Heimat' zu leugnen.

Indem sie die Prekarität von ,Heimat' anhand so diverser Themen nachvollzieht, trägt diese Sondernummer der Kulturwissenschaftlichen Zeitschrift dazu bei, dem Streit um Abschaffung oder Rehabilitation des Ausdrucks ein Drittes entgegen zu setzen: ,Heimat' als immer schon prekär zu betrachten kann, so sind wir überzeugt, dazu beitragen, nicht nur die geschichtlichen 
Formen der Begeisterung für und Ablehnung von ,natürlicher Zugehörigkeit zusammen zu führen, sondern den Ausdruck seiner normativen Gewalt zu berauben oder diese allerwenigstens in ihrer Selbstverständlichkeit zu stören. Die Herausgeber*innen danken allen Autor*innen für ihre Bereitschaft, sich auf die Fragen einzulassen und für ihre wertvollen Beiträge zu einer allzu oft heiß geführten Diskussion. Dank gilt auch Julie Mrosla, Robin Gieseke und Benjamin Wolf, die die Einrichtung der Beiträge in Dresden unterstützt haben.

\section{Literaturverzeichnis}

Aydemir, Fatma/Yaghoobifarah, Hengameh (Hg.) (2019): Eure Heimat ist unser Albtraum. Berlin: Ullstein fünf.

Bauman, Zygmunt (2006): Verworfenes Leben. Die Ausgegrenzten der Moderne. Mit Unterstützung von Werner Roller. 2. Auflage. Hamburg: Hamburger Edition.

Bauman, Zygmunt (2013): Liquid Fear. Cambridge: Polity. Brown, Wendy (2010): Walled States, Waning Sovereignty. New York, NY: Zone Books.

Costadura, Edoardo/Ries, Klaus/Wiesenfeldt, Christiane (2019): Heimat global: Einleitung. In: Dies. (Hg.) (2019): Heimat global. Modelle, Praxen und Medien der Heimatkonstruktion. 1. Auflage. Bielefeld: transcript, S. 11-44.

Cronon, William (1996): Trouble with Wilderness: Or, Getting Back to the Wrong Nature. In: Environmental History 1/1, 7-28. http://www.jstor.org/ stable/3985059 (letzter Zugriff 5.03.2020).

Gebhard, Gunther/Geisler, Oliver/Schröter, Steffen (Hg.) (2007): Heimat. Konturen und Konjunkturen eines umstrittenen Konzepts. Bielefeld: transcript Verlag (Kultur- und Medientheorie).

Günzel, Stephan: Heimat und Raum. Die Herkunft des Ortsprimats im Raumdiskurs aus der Heimatkunde, in: Bauer, Jenny; Gremler, Claudia; Penke, Niels (Hg.) (2014): Heimat, Räume. Komparatistische Perspektiven auf Herkunftsnarrative. Georg-AugustUniversität Göttingen; Nachwuchskonferenz „Heimat - Räume". 1. Aufl. Berlin: Bachmann (Studia Comparatistica, 3), S. 27-44.

Heinrich, Bernd (2014): The Homing Instinct. The Story and Science of Migration. London: William Collins.

Heinrich, Bernd (2016): Der Heimatinstinkt. Das Geheimnis der Tierwanderung. Mit Unterstützung von Kober. 1. Auflage. Berlin: Matthes \& Seitz (Naturkunden, 30).

Koch, Lars (2013): Lachen verbindet - transkulturelle Brückenschläge in der Culture-Clash-Comedy Almanya. In: Ehrhardt, Claus/Neuland, Eva: Der Deutschunterricht 4: Witze. Seelze: Friedrich in Velber, S. 36-47.

Koch, Lars/Nanz, Tobias/Pause, Johannes (2016): Imaginationen der Störung. Ein analytisches
Modell. In: Behemoth. A Journal on Civilization. 8/1: Imaginationen der Störung, S. 6-23.

Konzeptgruppe „Invektivität": Ellerbrock, Dagmar/Koch, Lars/Müller-Mall, Sabine/Münkler, Marina/Scharloth, Joachim/Schrage, Dominik/Schwerhoff, Gerd (2017): Invektivität - Perspektiven eines neuen Forschungsprogramms in den Kultur- und Sozialwissenschaften. In: Kulturwissenschaftliche Zeitschrift 2/1, S. 2-24. DOI: $10.2478 / \mathrm{kwg}-2017-0001$.

Menasse, Robert (2014): Heimat ist die schönste Utopie. Reden (wir) über Europa. Orig.-Ausg., 3. Aufl. Berlin: Suhrkamp (Edition Suhrkamp, 2689).

Mrozek, Bodo (2019): Von Anywheres und Somewheres. Das „Heimatbedürfnis der einfachen Menschen ist ein ahistorisches Konstrukt. In: Merkur 73/843, S. 32-47.

Nassehi, Armin: Woher kommst Du nicht? Sieben Exkursionen in eine Soziologie der Heimat, in: Nassehi, Armin/Felixberger, Peter/Schultheiß, Evelin (Hg.) (2019): Heimatt. Hamburg: Kursbuch Kulturstifung (Kursbuch, 198). E-Book.

Nell, Werner (2019): Heimatdiskurse und Gewalt. In: Costadura, Edoardo/Ries, Klaus/Wiesenfeldt, Christiane (Hg.) (2019): Heimat global. Modelle, Praxen und Medien der Heimatkonstruktion. 1. Aufl. Bielefeld: transcript, S. 105-132.

Nitzke, Solvejg (2018a): Prekäre Natur - Schauplätze ökologischen Erzählens zwischen 1840 und 1915. Eine Forschungsskizze. In: Kulturwissenschaftliche Zeitschrift 3/2, S. 31-48. DOI: 10.2478/ kwg-2018-0012.

Nitzke, Solvejg (2018b): Zurück zur Natur. Erfindung und Verschwinden der „Waldheimat" bei Peter Rosegger. In: Ehrler, Martin/Weiland, Marc (Hg.): Topografische Leerstellen. Ästhetisierungen verschwindender und verschwundener Dörfer und Landschaften (Rurale Topografien), S. 199-214.

Nitzke, Solvejg (2020): Walden Inc. Die letzten Männer. In: Merkur 848/74, S. 61-69.

Penke, Niels (2014): Die ,Urheimat der Germanen' und Lebensraum im Süden. Zur Extension von Ursprung und Heimat in völkischen Romanen (Blunck, Grimm, Vesper). In: Bauer, Jenny/Gremler, Claudia/Penke, Niels (Hg.) (2014): Heimat, Räume. Komparatistische Perspektiven auf Herkunftsnarrative. Georg-AugustUniversität Göttingen; Nachwuchskonferenz "Heimat - Räume". 1. Aufl. Berlin: Bachmann (Studia Comparatistica, 3), S. 109-122.

Rosa, Hartmut (2019): Heimat als anverwandelter Weltausschnitt. Ein resonanztheoretischer Versuch. In: Costadura, Edoardo/Ries, Klaus/Wiesenfeldt, Christiane (Hg.) (2019): Heimat global. Modelle, Praxen und Medien der Heimatkonstruktion. 1. Aufl. Bielefeld: transcript, S. 153-172.

Scharnowski, Susanne (2016): Die Wiederkehr und das Verschwinden der Heimat. Das Landleben in der Gegenwartsliteratur, in: Hellström, Martin/Karlsson Hammarfelt, Linda/Platen, Edgar (Hg.): Umwelt sozial, kulturell, ökologisch. Zur Darstellung von Zeitgeschichte in deutschsprachiger Gegenwartsliteratur. München: iuducium, S. 10-24. 
Scharnowski, Susanne (2019a): Heimat. Geschichte eines Missverständnisses. Darmstadt: wbg.

Scharnowski, Susanne (2019b): „Das große Missverständnis", DLF Interview vom 6.10.2019 URL: https://www. deutschlandfunk.de/heimatbegriff-in-der-kulturgeschichte-das-grosse.1184. de.html?dram:article_id=457936 (letzter Aufruf 04.04.2020).
Schütz, Erhard (2019a): Vom Walde her und in die Wälder. In: Ders.: Mediendiktatur Nationalsozialismus. Heidelberg: Winterverlag. (Siegen/Beiträge zur Literatur-, Sprach- und Medienwissenschaft, 179), S. $85-152$.

Sebald, W. G. ([1995] 2012): Unheimliche Heimat. Essays zur österreichischen Literatur. 4. Aufl. Frankfurt am Main: Fischer-Taschenbuch-Verl. (Fischer, 12150). Stanišić, Saša (2019): Herkunft. München: Luchterhand. 